\title{
Evaluation of the efficacy of a new hyaluronic acid gel on dynamic and static wrinkles in volunteers with moderate aging/photoaging
}

This article was published in the following Dove Medical Press journal: Clinical, Cosmetic and Investigational Dermatology

\author{
Adele Sparavigna' \\ Beatrice Tenconi' \\ Andrea Maria Giori \\ Gilberto Bellia ${ }^{3}$ \\ Laura La Penna' \\ 'DERMING S.r.l., Clinical Research \\ and Bioengineering Institute, Milan \\ Italy; ${ }^{2}$ Research and Development, \\ IBSA Farmaceutici Italia, Lodi, Italy; \\ ${ }^{3}$ Dermoaesthetic Business Unit, IBSA \\ Farmaceutici Italia, Lodi, Italy
}

Purpose: Aim of the study was to determine both clinically and by noninvasive instrumental evaluations the efficacy, tolerability and the duration of the effects of a new hyaluronic acid (HA) gel in human volunteers with moderate aging/photoaging.

Patients and methods: Eighteen volunteers (35-55 years) were enrolled in this single-center study. The subjects underwent five visits. The first visit was at baseline to determine the adherence to the inclusion criteria, followed by the first injection of the HA-based study product, and the second visit was at 48 hours after the injection. Two months later, a second injection was given (Visit 3) followed by a subsequent visit (Visit 4) after 48 hours. The last visit (Visit 5) was performed 5 months after the first injection. Clinical and instrumental evaluations as well as self-assessment by the subjects were recorded at each visit.

Results: A significant improvement of wrinkles' grade around the eyes, vertical lip lines and wrinkles' severity of nasolabial folds was recorded after the first injection and the effect increased after the second injection. Aging/photoaging grade and surface microrelief improved 2 months after the first injection procedure. These clinical improvements were paralleled by amelioration of instrumental skin profilometry and optical colorimetry. The treatments were very well tolerated by the volunteers as determined by the self-grading score.

Conclusion: The results confirm the good esthetic performance and the duration of the effect of the HA-based study product (Viscoderm ${ }^{\circledR}$ Hydrobooster) on dynamic facial wrinkles and/or static facial lines. These effects were particularly evident after the second injection and were accompanied by a good tolerability of the product.

Keywords: wrinkles, facial aging, clinical assessment, instrumental assessment

\section{Introduction}

The entire human body is subject to changes associated with aging. ${ }^{1,2}$ Those occurring in the skin, even if not life threatening, are visible, undesirable and therefore associated with discomfort. ${ }^{3}$ Facial aging can be particularly negative since it represents the main image we offer to the people we are in contact with. ${ }^{4}$ Among the factors playing an important role in facial aging are exposure to ultraviolet (UV) light, smoking habits and alcohol consumption, which, together with genetic factors, can alter the rate of skin aging. ${ }^{5-8}$ One of the major biological factors influencing skin aging is the loss of dermal proteins such as collagen and elastin (resulting in the modification of extracellular matrix), hyaluronic acid (HA) and proteoglycans (which reduce the amount of water in the epidermis). ${ }^{9,10}$ These changes lead to the formation of wrinkles, fine lines, color changes, dryness and decreased elasticity. Another important factor in skin aging is represented by oxidative
Correspondence: Adele Sparavigna DERMING S.r.I., Clinical Research and Bioengineering Institute, Via Valassina 24, Milano (MI), Italy

Tel +39223183475

Email adele.sparavigna@derming.com 
stress, which ultimately results in the formation of ROS and DNA adducts, which are additional negative factors. ${ }^{5,8}$

Since the life expectancy of the world population is progressively increasing, ${ }^{11}$ particularly in developed countries, there is an increased demand for treatments, either preventive or esthetic, aimed at improving the quality of aging. A relatively recent survey conducted in the USA by the American Society for Dermatologic Surgery indicated that roughly $70 \%$ of the interviewed persons were concerned with skin texture and/or sagging. ${ }^{12}$

Several options are available to prevent or treat skin aging, including surgery, skin resurfacing and the utilization of injectable dermal fillers. ${ }^{13-16} \mathrm{HA}$, in particular, is widely used thanks to its high biocompatibility (being a component of the extracellular matrix and being present in large amounts in human tissues) and its ability to reduce wrinkles (either superficial or deep) and general skin aging. ${ }^{17-19}$ Although several different dermal fillers contain HA, they differ in their physicochemical characteristics, and this can result in a different clinical efficacy. The present single study was designed to test the efficacy and tolerability, as well as the duration of action of the HA-based study product, in volunteers with moderate aging/photoaging.

The HA-based study product is a ready-to-use solution of stabilized, injectable HA which has unique rheological properties.

Rheological measurements were performed using a Modular Compact MCR302 Rheometer (Anton Paar, Ostfildern, Germany) equipped with parallel plate geometry $(50 \mathrm{~mm}$ plate diameter, $1.0 \mathrm{~mm}$ gap) and Peltier temperature control. Measurements were carried out at $37^{\circ} \mathrm{C}$. In particular, the linear viscoelastic range (LVR), the mechanical spectra, the storage modulus $\left(G^{\prime}\right)$ and the loss modulus $\left(G^{\prime \prime}\right)$ were determined. Amplitude sweep tests were performed at a constant oscillatory frequency of $1.59 \mathrm{~Hz}$, over a strain range of $0.01 \%-100 \%$. The LVR was determined as the range of stress values in which $G^{\prime}$ and $G^{\prime \prime}$ remain constant. Then $G^{\prime}$ and $\mathrm{G}^{\prime \prime}$ values were extrapolated at 0.2 Pa stress (within $L V R$ ):

$$
\begin{aligned}
& \mathrm{G}^{\prime}(\mathrm{Pa})=37 \pm 2 \\
& \mathrm{G}^{\prime \prime}(\mathrm{Pa})=20 \pm 1
\end{aligned}
$$

A representative graph of the rheological analysis performed is shown in Figure 1.

These rheological properties confirm the high deformability and low gel stiffness and viscosity, allowing rapid tissue integration and the possibility to use it at different dermis levels up to the most superficial one.

\section{Patients and methods}

This was an open, single-center, pilot study conducted on 18 female volunteers under the control of dermatologists. All the volunteers signed a consent form containing the information relative to the nature and procedures of the study. This study was conducted according to the ethics of the "Helsinki declaration". The information and data on the trial are generated, recorded, documented and processed in accordance with a specific procedure, based on The International Council for Harmonisation of Technical Requirements for Pharmaceuticals for Human Use (ICH) Good Clinical Practice (GCP) 1996.

The HA-based study product (Viscoderm ${ }^{\circledR}$ Hydrobooster) was used, depending on the severity of the wrinkles, as a microdroplet injection for less-severe (dynamic) wrinkles (using $<0.01 \mathrm{~mL}$ for injection), or with a combined microlinear retrograde technique followed by microdroplet injection (both with volumes $<0.01 \mathrm{~mL}$ ) for static wrinkles.

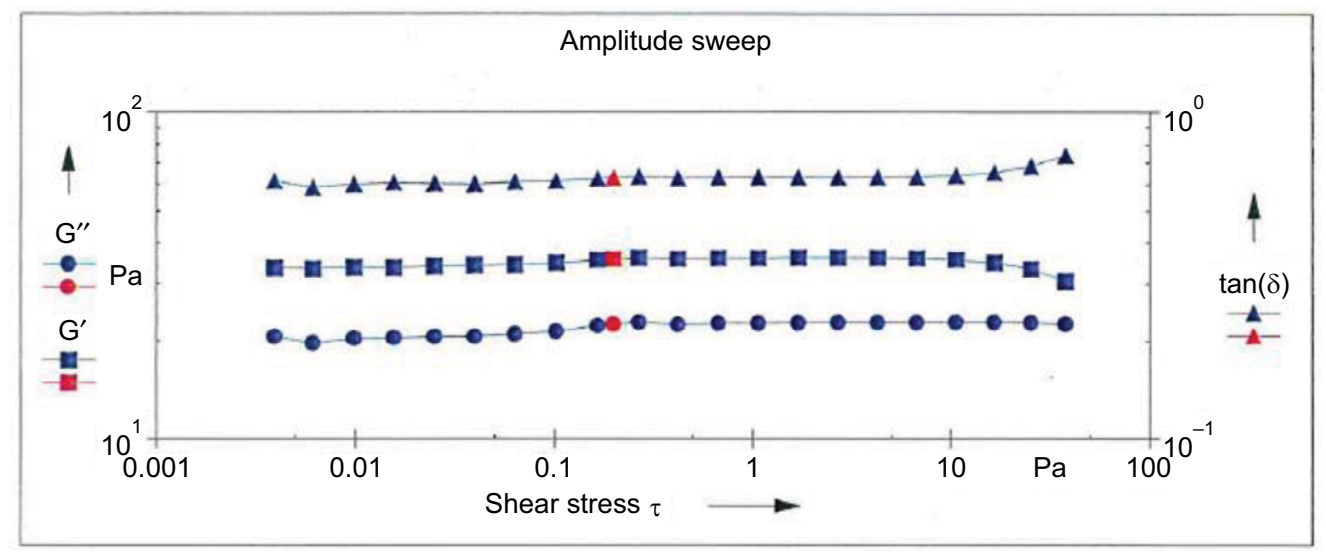

Figure I Amplitude sweep analysis in a representative batch of the HA-based study product. Abbreviation: HA, hyaluronic acid. 
The main inclusion criteria were: age between 35 and 55 years, moderate cutaneous aging/photoaging of grade 2-3 according to the Glogau's photographic scale, accepting to maintain their habits (food, physical activity, make-up use, facial cosmetics and cleansing products) and not to expose their face to strong UV irradiation without proper sun protection during the entire study period. The main exclusion criteria were pregnancy, lactation, smoking, alcohol or drug abuse, having received skin treatments for esthetic correction in 6 months prior to the study start date, having already performed permanent dermal fillers in the past, dermatological diseases (including dermatitis, cutaneous diseases in the tested area, recurrent facial and labial herpes) as well as general diseases such as diabetes, endocrine disease, hepatic, renal, cardiac and pulmonary disorders, cancer, neurological diseases, drug allergy and inflammatory or immunosuppressive diseases. Additional exclusion criteria were the use of anticoagulants and antiplatelet drugs, antihistamines, corticosteroids (both topic and systemic), narcotics, antidepressants, immunosuppressive drugs (a part of hormonal or contraceptive treatment which started $>1$ year before the study) and, in general, drugs potentially interfering with the results according to the opinion of the investigators.

\section{The study included five visits.}

1. First one at baseline (T0) with all the clinical and instrumental evaluations to check all the inclusion and exclusion criteria (immediately followed by the first injection procedure);

2. Next one at 48 hours after the first injection procedure (T1i);

3. Next one at 2 months after the first injection (T2, concomitantly starting the second injection);

4. Next one at 48 hours after the second injection (T2i) and

5. The last one (T5) after 5 months from the first injection.
The injections were given, according to the product leaflet, using two different techniques:

1. A microbolus technique for the dynamic facial wrinkles (microdroplet injections $<0.01 \mathrm{~mL}$ each along the path of the wrinkle) and

2. A combined technique for the static facial wrinkles (microlinear retrograde technique $<0.01 \mathrm{~mL}$ each below the path of the wrinkle, followed by microdroplet injections $<0.01 \mathrm{~mL}$ on the same wrinkle).

These techniques reduce and prevent the appearance of wrinkles and fine lines and preserve and restore the quality of the skin maintaining its elasticity, improving the thickness, firmness and hydration.

Evaluation of the efficacy of the treatment was clinical (qualitative) and based on instrumental measurements (quantitative), and was performed at each study visit.

The clinical evaluation relative to aging and photoaging was based on the visual score of deep and fine wrinkles according to the Glogau's reference scale which goes from 0 (no wrinkles) to 4 (only wrinkles with severe photoaging), as shown in Table 1. The clinical evaluation of the surface microrelief was based on the Beagley Gibson reference scale, which again spans from 1 to 4 (Table 1).

A photographic record (both static and dynamic) of the face was taken using standardized procedures to allow comparable evaluation of the images.

As for the instrumental evaluations, these were taken monolaterally on the cheek at all five visits of the study. The different measures were performed under standard environmental conditions, and the volunteers were acclimatized for 10-15 minutes before each measurement.

The instrumental procedures included: optical colorimetry, using a tri-stimulus colorimeter (Chroma Meter CR-200) equipped with three special filters, which determines skin

Table I Glogau and Beagley Gibson scores used for clinical evaluation of the efficacy

\begin{tabular}{|l|l|l|}
\hline Score & Glogau scale & Beagley Gibson scale \\
\hline I & $\begin{array}{l}\text { Early photoaging, mild pigment changes, minimal wrinkles, no "age } \\
\text { spots" }\end{array}$ & $\begin{array}{l}\text { Primary lines are all at the same depth; secondary lines are } \\
\text { well demarcated, forming star-like pictures }\end{array}$ \\
\hline 2 & $\begin{array}{l}\text { Early to moderate photoaging, appearance of line only when the face } \\
\text { moves, early brown "age spots", prominent skin pores, early changes } \\
\text { in skin texture }\end{array}$ & $\begin{array}{l}\text { Hiding and loss of secondary lines demarcation. Star-like } \\
\text { pictures present, but with less-demarcated secondary lines }\end{array}$ \\
\hline 3 & $\begin{array}{l}\text { Advanced photoaging, prominent brown pigmentation, visible brown } \\
\text { "age spots", prominent and small blood vessels, wrinkles present } \\
\text { with face at rest }\end{array}$ & $\begin{array}{l}\text { Irregular primary lines, strong hiding of secondary lines with } \\
\text { low star-like pictures }\end{array}$ \\
\hline 4 & $\begin{array}{l}\text { Severe photoaging, diffused wrinkles, yellow-gray skin color, prior } \\
\text { skin cancer, actinic keratosis }\end{array}$ & $\begin{array}{l}\text { Strong skin deterioration, distortion of and loss of secondary } \\
\text { lines, deep primary lines }\end{array}$ \\
\hline
\end{tabular}


brightness; wrinkle pictures and profilometry using a Primos compact portable device (GFMesstechnik, Teltow, Germany) which measures and elaborates, thanks to a dedicated software, skin wrinkles and major skin profilometric parameters both in vivo and in skin replicas.

A self-grading evaluation was performed by each participant after both injections, using a visual analogic scale (VAS; 10 units in length). The scale recorded all the major unpleasant sensations related to the injections, such as stinging, itching, tightening, burning, pain and discomfort.

The investigators checked for any immediate local reaction (tardive swelling, pain, erythema, bruising) as well as any additional adverse event, including the systemic ones.

\section{Statistical analysis}

Statistical analysis was performed using different tests as follows:

1. For clinical assessment, the Friedman test was performed, which was followed, in case of statistically significant results, by the Holm-Sidak Adjusted test.

2. For the data arising from the instrumental evaluations, a non-parametric test (Friedman test) was used when the normality hypothesis was rejected by the Shapiro-Wilk normality test (with the threshold set at 5\%). When the normality hypothesis was confirmed, a parametric test (ANOVA test for repeated measures) was used, followed by the Holm-Sidak Adjusted test when the results were statistically significant.

For the VAS scores, a non-parametric test (Wilcoxon test) was used.

\section{Results}

Of the 18 volunteers enrolled, 14 completed the entire study, while 4 participants prematurely stopped the trial for personal reasons not related with the study. No additional events potentially interfering with the results occurred during the study period.

\section{Clinical assessment}

Forty-eight hours after the first injection (T1i), a statistically significant improvement of the wrinkles grade around the eyes $(23.1 \%)$ was recorded. This improvement increased at the subsequent evaluation points $(38.5 \%$ at $\mathrm{T} 2,43.6 \%$ at T2i and 35.9 at T5) with a statistically significant difference (Holm-Sidak Adjusted Wilcoxon rank test $P<0.05$ for all the time points relative to the baseline [T0]), as shown in
Figure 2A. This improvement corresponded to a reduction of the clinical score of 1 grade for $86 \%$ of the subjects at $\mathrm{T} 1 \mathrm{i}$ and for all the 14 volunteers at all the other time points. In Figure 2B, representative facial images showing the area around the eyes at baseline (T0) and 5 months after the first injection (T5) are shown. As it can be seen, there is a clear improvement in the wrinkles present at baseline.

With a similar trend, there was a statistically significant reduction (Holm-Sidak Adjusted Wilcoxon signed rank test $P<0.05$ at all the study visits relative to baseline) of vertical lip lines (Figure 3), which was of $29.2 \%$ at T1i, $41.7 \%$ at $\mathrm{T} 2,50 \%$ at $\mathrm{T} 2 \mathrm{i}$ and $41.7 \%$ at $\mathrm{T} 5$. This reduction again corresponded to a decrease in the clinical score of at least 1 grade, which was achieved by $64 \%$ of the subjects at T1i, $86 \%$ at $\mathrm{T} 2$ and $\mathrm{T} 2 \mathrm{i}$ and $79 \%$ at $\mathrm{T} 5$.

Concomitantly, a statistically significant improvement (Holm-Sidak Adjusted Wilcoxon signed rank test $P<0.05$ for T2, T2 i and T5 relative to baseline) of wrinkle severity of nasolabial folds was obtained, which was $14.7 \%$ at T1i and $29.4 \%$ at all the other study visits (Figure 4). The Holm-Sidak Adjusted Wilcoxon rank test reported a value of $P<0.05$ for $\mathrm{T} 2, \mathrm{~T} 2 \mathrm{i}$ and $\mathrm{T} 5$ relative to the baseline (T0). A reduction of the clinical score of at least 1 grade was achieved by $43 \%$ of subjects at T1i, 93\% at T2, 93\% at T2 $\mathrm{i}$ and $86 \%$ at T5.

A statistically significant improvement of the aging/photoaging grade was recorded starting from T2. A reduction of $8 \%$ of the Glogau's score mean value was determined at $\mathrm{T} 2$, $12 \%$ at T2 $\mathrm{i}$ and $8 \%$ at T5 (Figure 5A). Also, for the surface microrelief (Figure 5B), a statistically significant improvement (Holm-Sidak Adjusted Wilcoxon signed rank test $P<0.05$ for $\mathrm{T} 2$, $\mathrm{T} 2 \mathrm{i}$ and $\mathrm{T} 5$ relative to baseline) was obtained starting from $\mathrm{T} 2$, which accounted for $23.3 \%$ at T2 and $26.7 \%$ at all the other study visits. This reduction corresponded to a decrease of at least 1 grade of the clinical score in $71 \%$ of the subjects already at $\mathrm{T} 2$.

\section{Instrumental evaluation}

Data on skin profilometry indicate an important and clinically relevant anti-wrinkle activity in the area around the eyes. Figure 6 shows that there was a reduction (relative to baseline) of the parameters $\mathrm{Ra}$ (average roughness of the analyzed profile, Holm-Sidak Adjusted test $P<0.05$ for T2, T2 i and T5 relative to baseline), which indicates a less-wrinkled area, Rt (wrinkles' total height, Holm-Sidak Adjusted test $P<0.05$ for $\mathrm{T} 2 \mathrm{i}$ and $\mathrm{T} 5$ relative to baseline), which indicates the presence of less-marked wrinkles, and Rv (wrinkles' maximum depth, Holm-Sidak Adjusted test $P<0.05$ for T5 relative to baseline), indicating that the wrinkles were less deep. 
A

Wrinkles' grade around the eyes

(Crow's feet reference photographic scale)

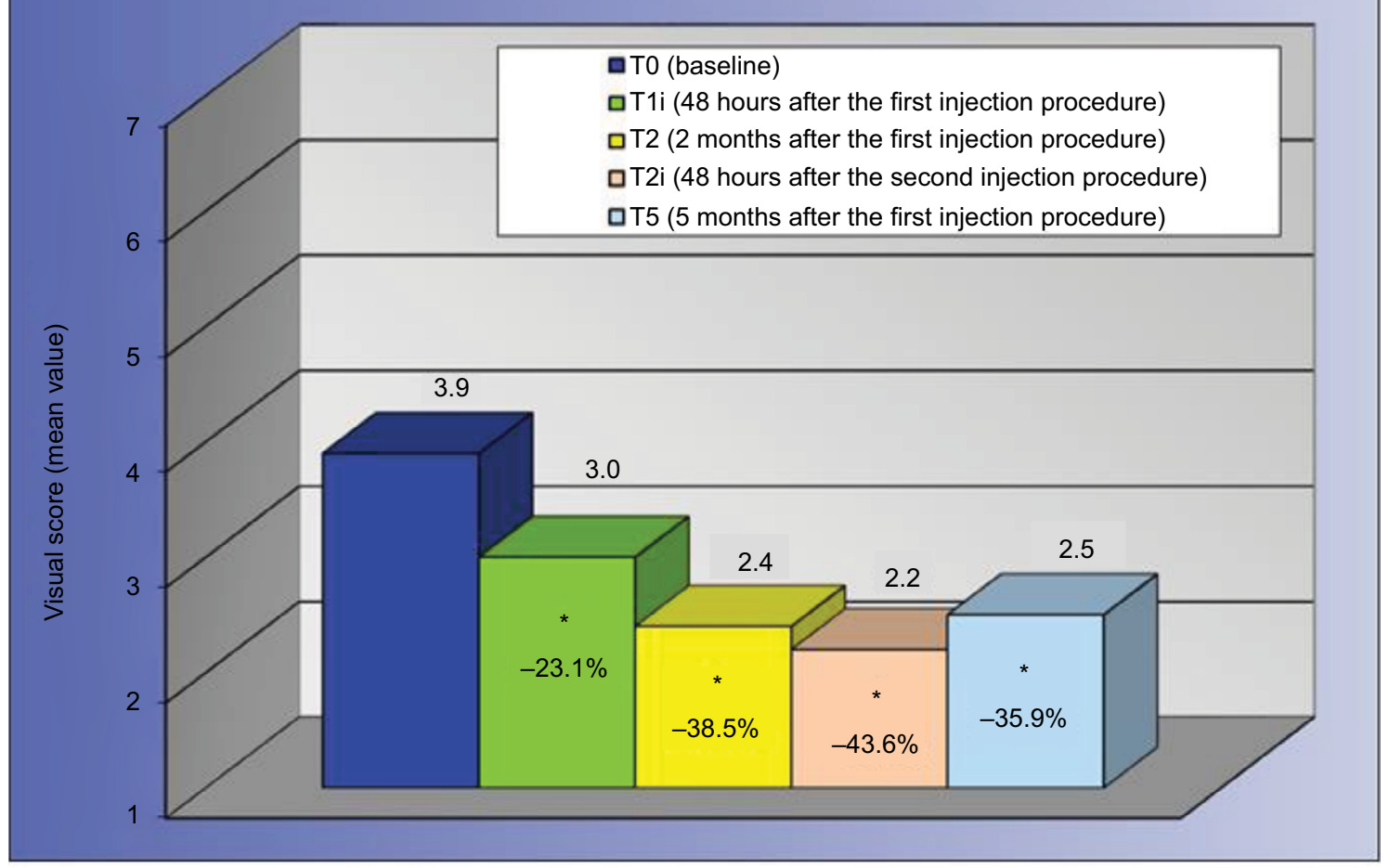

B
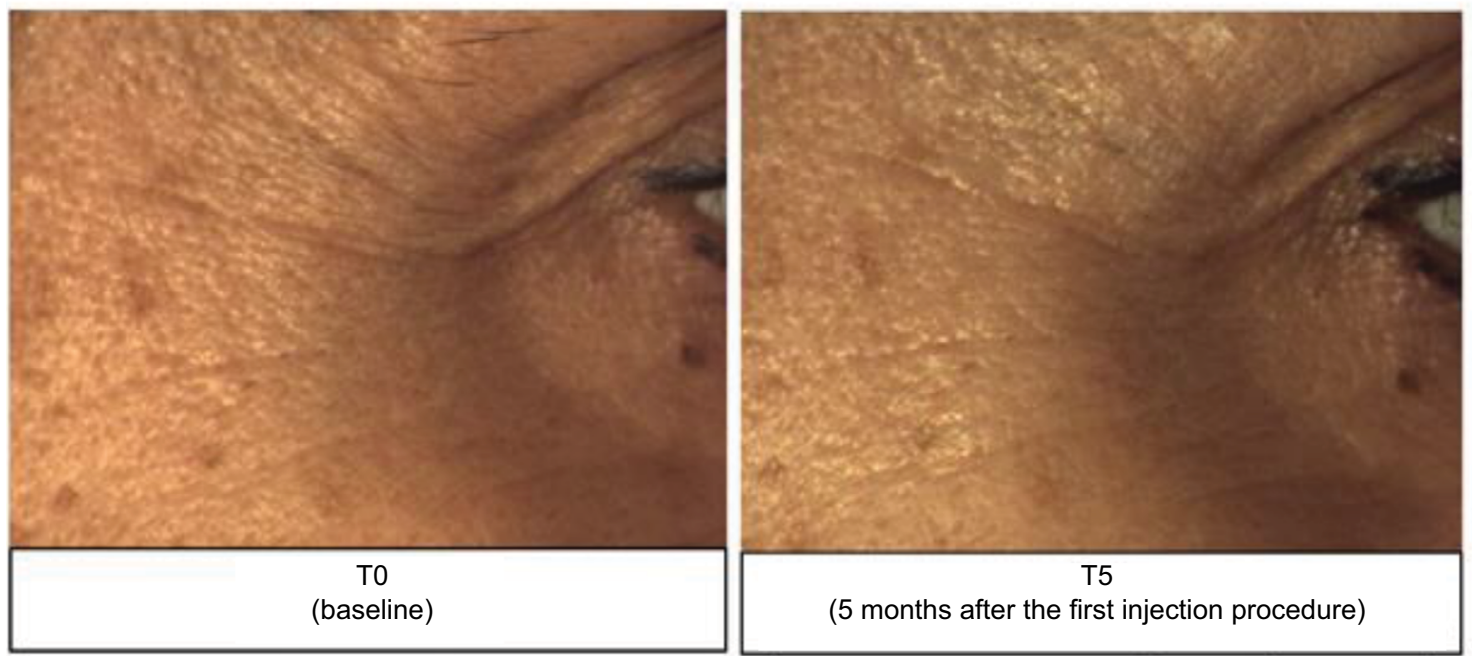

Figure 2 Evaluation of wrinkles around the eyes.

Notes: (A) Clinical evaluation of wrinkle grade around the eyes at the different time points. ${ }^{*}<0.05$ vs T0 (Holm-Sidak Adjusted Wilcoxon signed rank test). (B) Representative images of the area around the eyes before and after treatment. 


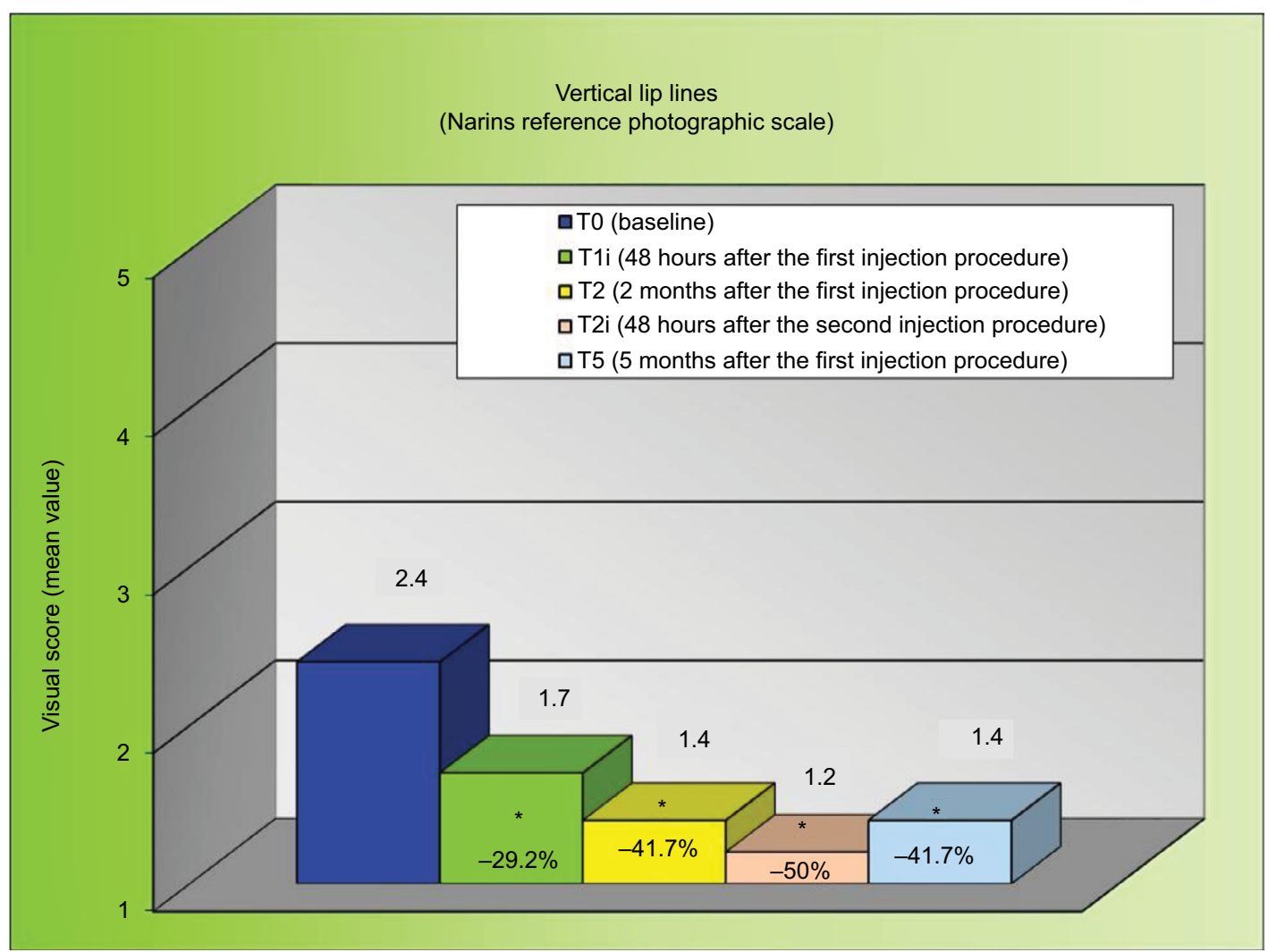

Figure 3 Clinical evaluation of vertical lip lines at the different time points. Note: $* P<0.05$ vs T0 (Holm-Sidak Adjusted Wilcoxon signed rank test).

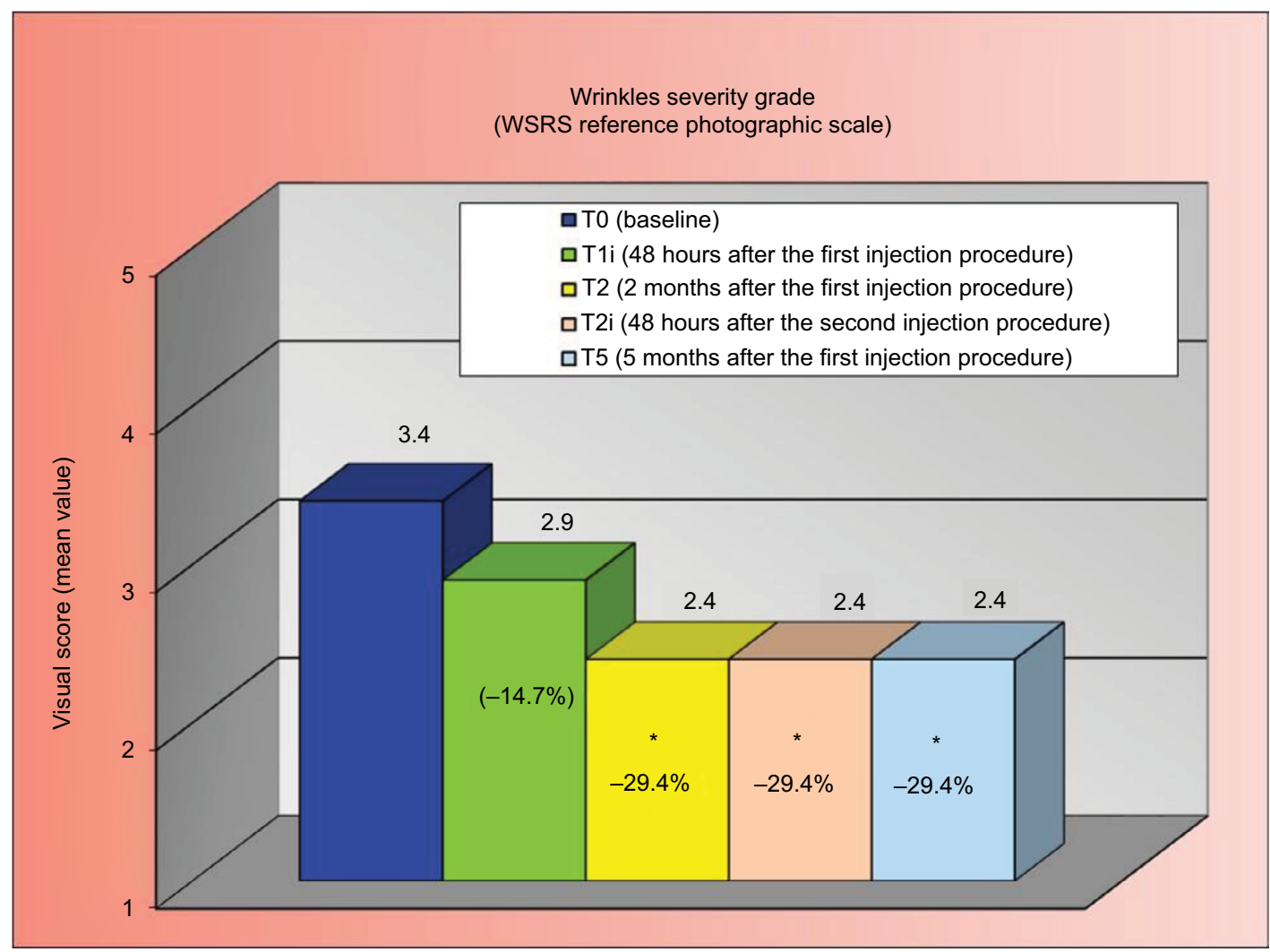

Figure 4 Clinical evaluation of wrinkles' severity grade at the different time points.

Note: $* P<0.05$ vs T0 (Holm-Sidak Adjusted Wilcoxon signed rank test).

Abbreviation: WSRS, Wrinkle Severity Rating Scale. 
A

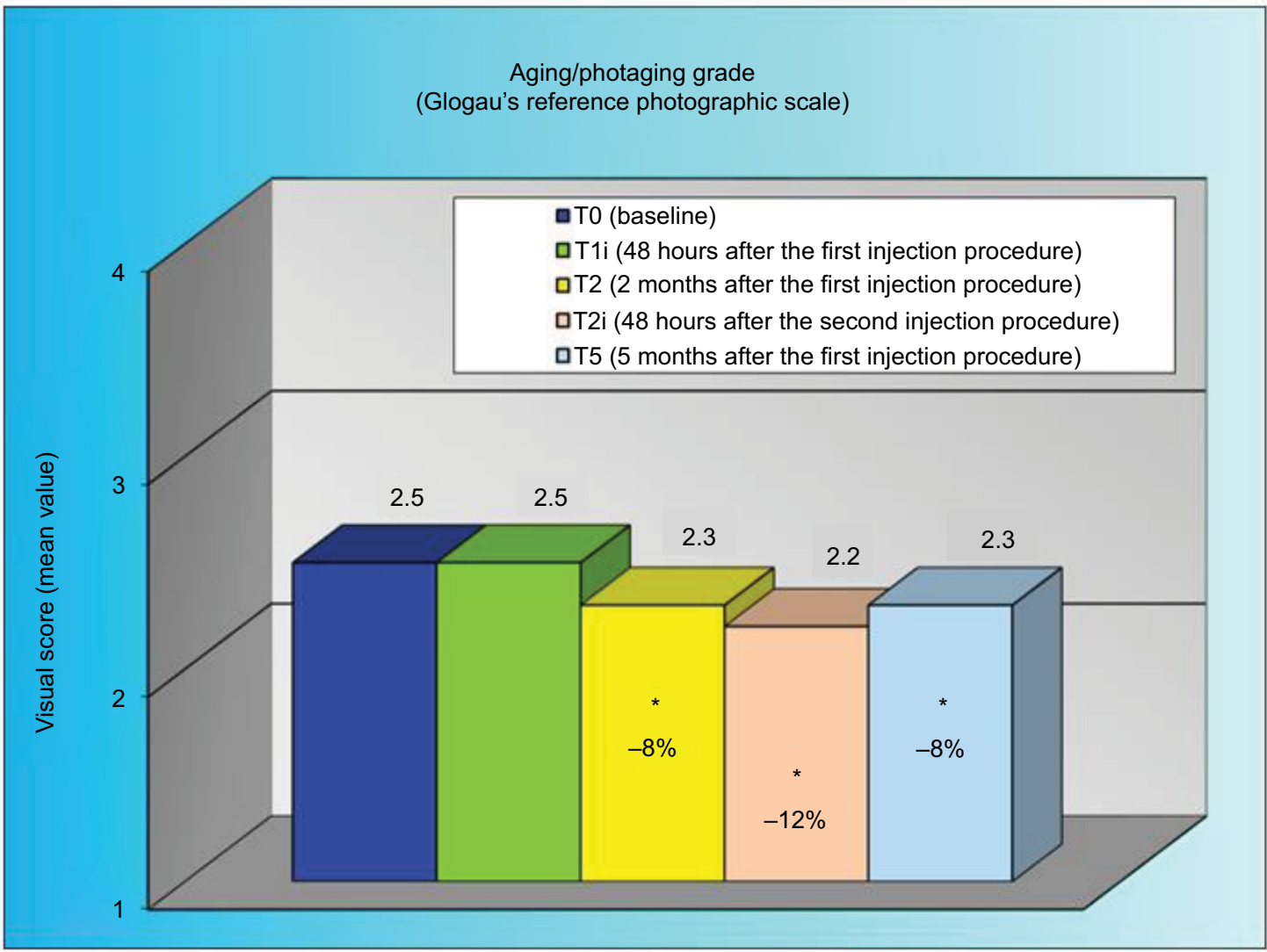

B

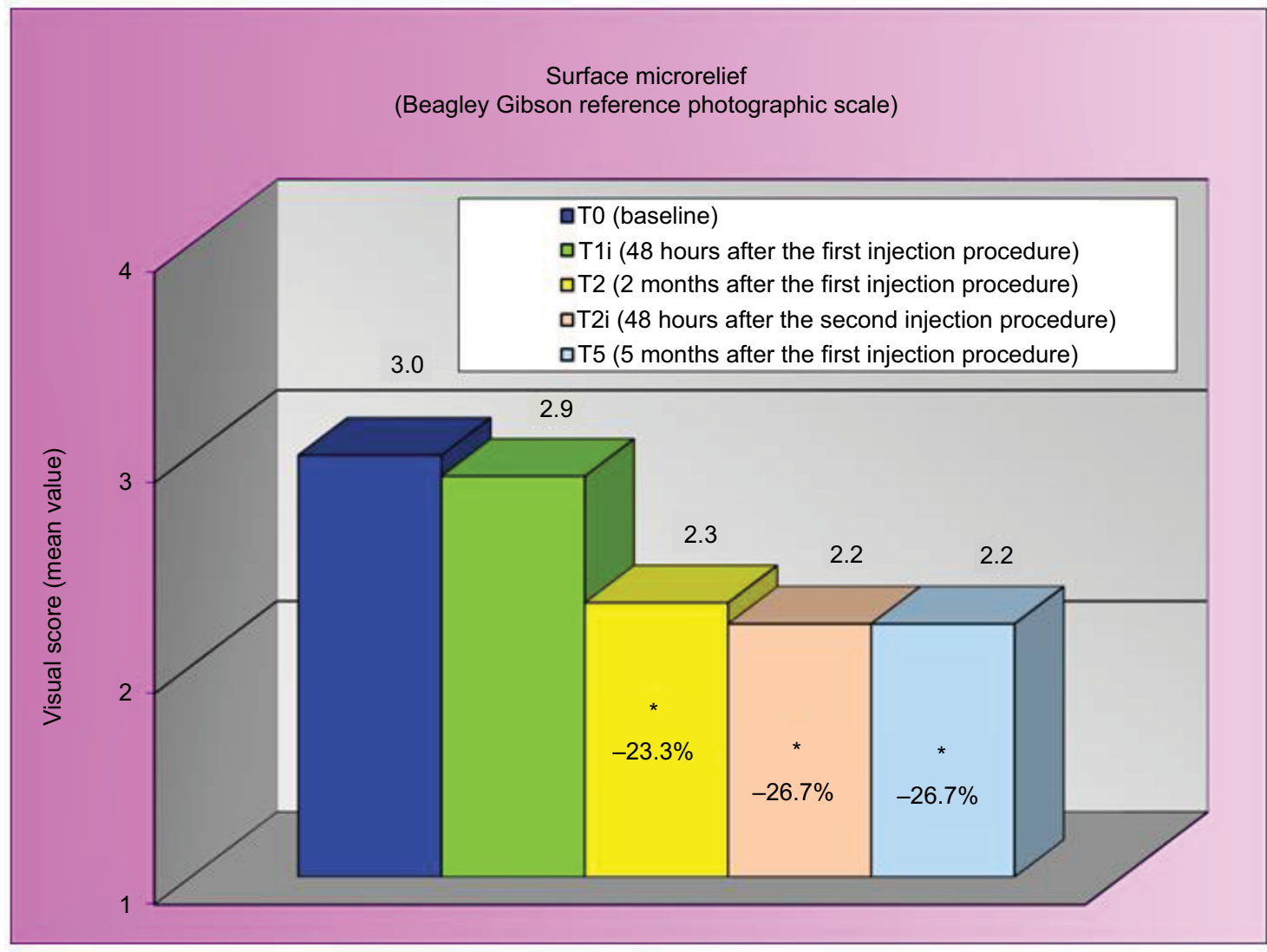

Figure 5 Clinical evaluation of aging/photoaging grade (A) and surface microrelief (B) at the different time points.

Note: ${ }^{*}<<0.05$ vs T0 (Holm-Sidak Adjusted Wilcoxon signed rank test). 
A

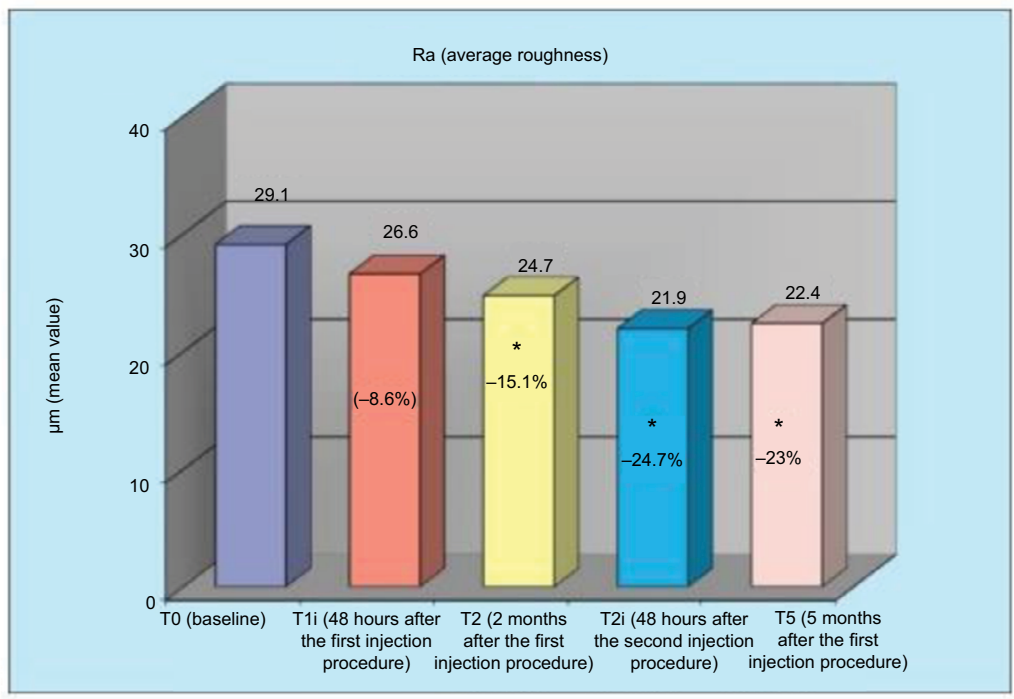

B

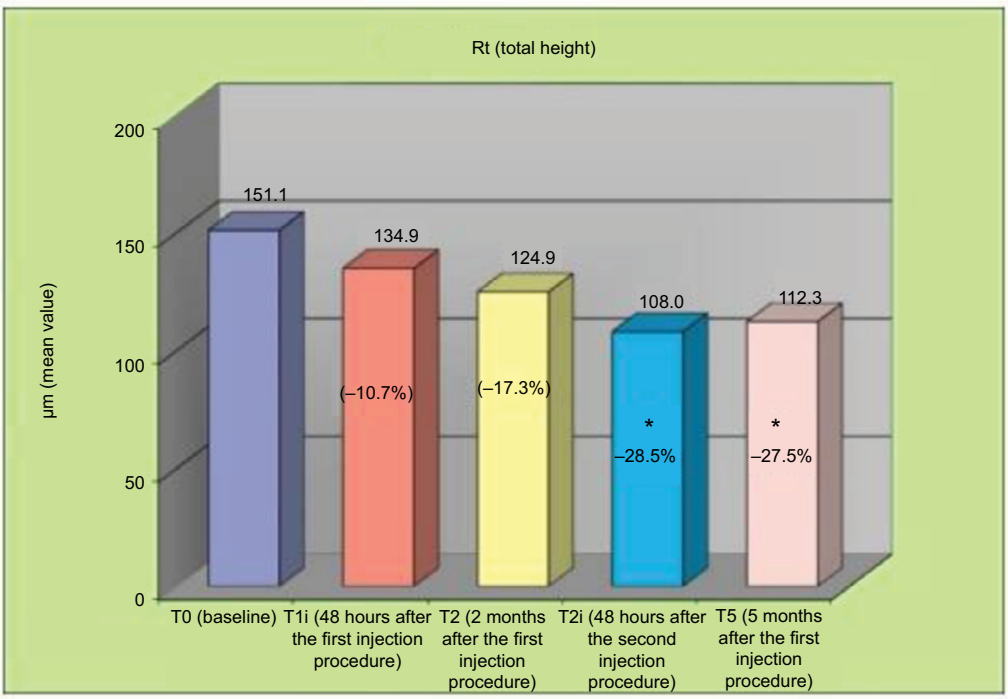

c

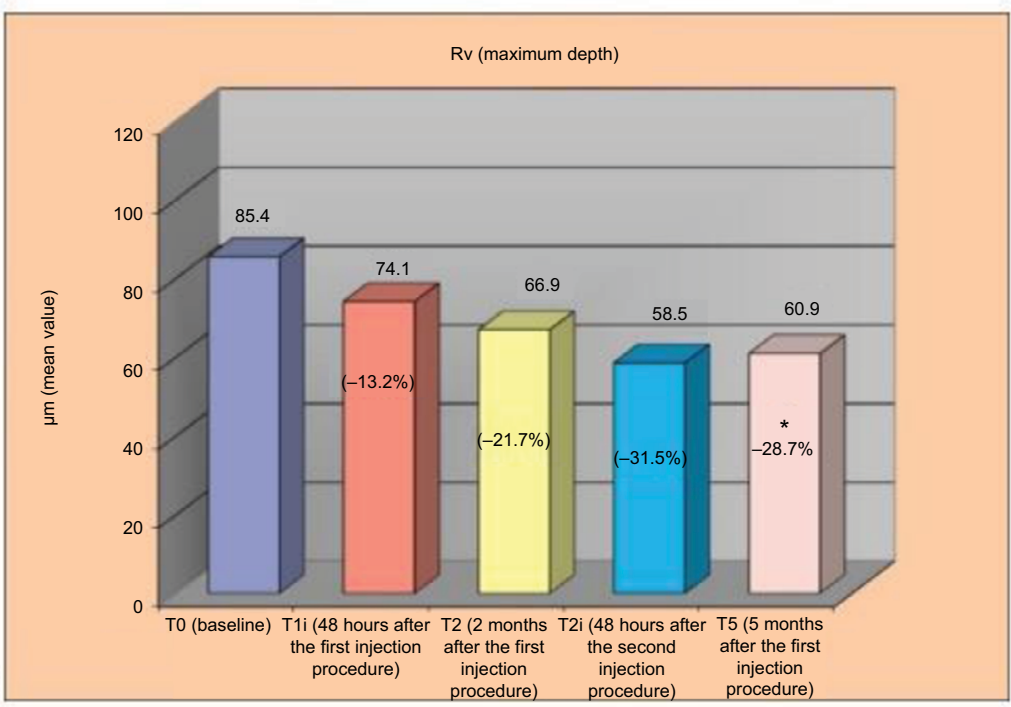

Figure $\mathbf{6}$ Skin profilometry of the area around the eyes reporting $(\mathbf{A})$ average roughness $\mathrm{Ra}$, $(\mathbf{B})$ total height $\mathrm{Rt}$ and $(\mathbf{C})$ maximum depth $\mathrm{Rv}$ at the different time points. Note: ${ }^{*} P<0.05$ vs T0 (Holm-Sidak Adjusted $t$-test/Wilcoxon signed rank test). 
Using optical colorimetry, a statistically significant improvement of $\mathrm{L}^{*}$ parameter, representing skin brightness, was achieved starting from T2 (Figure 7).

\section{Tolerability}

The self-assessment of the subjects' sensations, recorded immediately after the first and second injections, is reported in Table 2. The table reports the percentage of volunteers reporting a VAS value $>5$. As it can be seen, the percentage of patients reporting a score $>5$ was low for pain and skin discomfort that represent the most unpleasant sensations, particularly experienced after the second injection.

\section{Discussion}

HA represents the most widely used dermal filler for the treatment of skin aging. ${ }^{17}$ It has demonstrated clinical efficacy in the treatment of superficial and deep wrinkles and in reducing wrinkle severity., ${ }^{9,14,18,19}$ It represents an ideal compound to be used as a dermal filler thanks to its tolerability, biocompatibility and lack of allergic reactions (being a component of the extracellular matrix and present in elevated amounts in different tissues). All these characteristics have prompted the generation of several HA preparations. One of the most important characteristics of a dermal filler is its ability not only to ameliorate the skin aging process but also to maintain its efficacy for sufficient time. ${ }^{19-22}$ Concomitantly, it must have a safe profile with reduced distress.

To test the efficacy, duration and tolerability of the HA study product, clinical and instrumental evaluations, together with a subject self-evaluation, have been used. Interestingly,

Table 2 Percentage of subjects reporting a VAS score greater than 5

\begin{tabular}{|l|l|l|}
\hline \multirow{2}{*}{$\begin{array}{l}\text { Unpleasant } \\
\text { sensation }\end{array}$} & \multicolumn{2}{|l|}{$\%$ of subjects (n) } \\
\cline { 2 - 3 } & $\begin{array}{l}\text { Immediately after } \\
\text { the first injection }\end{array}$ & $\begin{array}{l}\text { Immediately after } \\
\text { the second injection }\end{array}$ \\
\hline Stinging & $14(2)$ & $\mathrm{I}(2)$ \\
\hline Itching & $7(\mathrm{I})$ & $\mathrm{I}(2)$ \\
\hline Burning & $2 \mathrm{I}(3)$ & $2 \mathrm{I}(3)$ \\
\hline Tightening & $7(\mathrm{I})$ & $\mathrm{I}(2)$ \\
\hline Pain & $29(4)$ & $36(5)$ \\
\hline Discomfort & $14(2)$ & $29(4)$ \\
\hline
\end{tabular}

Abbreviation: VAS, visual analog scale.

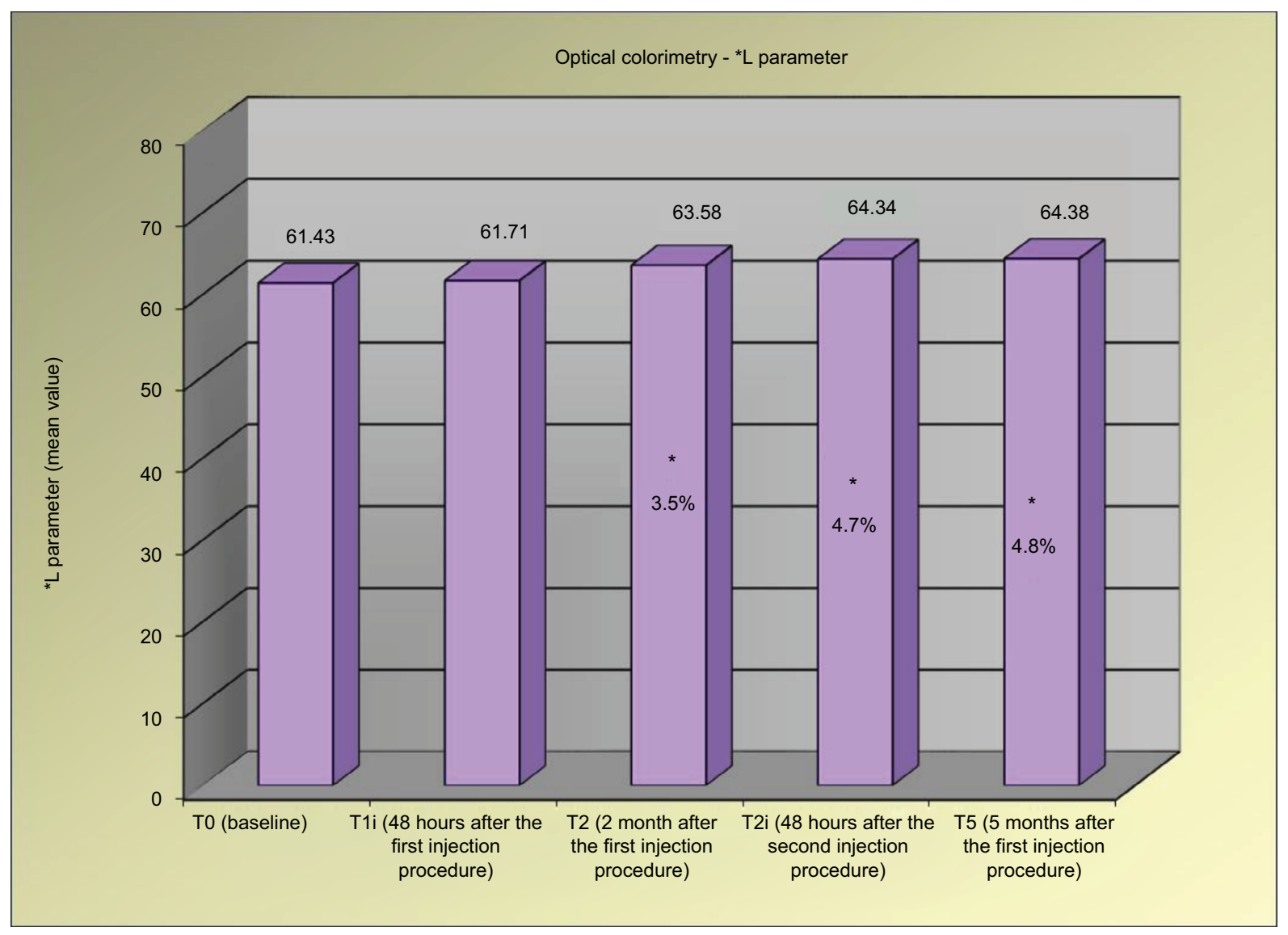

Figure $\mathbf{7} \mathrm{L} *$ Parameter determined by optical colorimetry at the different time points. Note: $* P<0.05$ vs T0 (Holm-Sidak Adjusted $t$-test). 
for all the parameters evaluated, both clinical and instrumental evaluations were concordant in defining the clinical efficacy of the product in the treatment of facial wrinkles. Notably, the study was planned to determine the immediate effects and those lasting 5 months after the initial administration, and the results not only indicated a rapid amelioration of the parameters but also showed that these effects were even increased after the second injection and maintained for the entire period of the study. This is a particularly relevant finding, which suggests that the use of a limited number of procedures can be sufficient to exert a clinically and statistically significant effect. Since the intradermal injection is not free from unpleasant effects, the possibility of producing a long-lasting effect with limited injections is certainly a plus for any HA-containing filler. The self-evaluation of the volunteers confirmed the tolerability of the product, which showed only a few unpleasant effects.

\section{Conclusion}

The results of this single-center study demonstrated the clinical efficacy (in all the efficacy tests performed) and good tolerability of the HA-based study product (Viscoderm Hydrobooster). A particular advantage is the ability of the product to maintain its efficacy for a long time, thus helping to reduce the number of intradermal injections.

\section{Acknowledgment}

The authors are grateful to Giovanna Damia for the help in writing the manuscript. This study has been sponsored by IBSA Farmaceutici Italia.

\section{Disclosure}

AMG and GB are employees of IBSA Farmaceutici Italia. AS, BT and LLP are employees of DERMING S.r.1. The authors report no other conflicts of interest in this work.

\section{References}

1. Khan SS, Singer BD, Vaughan DE. Molecular and physiological manifestations and measurement of aging in humans. Aging Cell. 2017;16(4):624-633.

2. Kazanci A, Kurus M, Atasever A. Analyses of changes on skin by aging. Skin Res Technol. 2017;23(1):48-60.
3. Charles Finn J, Cox SE, Earl ML. Social implications of hyperfunctional facial lines. Dermatol Surg. 2003;29(5):450-455.

4. Dayan SH, Lieberman ED, Thakkar NN, Larimer KA, Anstead A. Botulinum toxin a can positively impact first impression. Dermatol Surg. 2008;34(Suppl 1):S40-S47.

5. McDaniel D, Farris P, Valacchi G. Atmospheric skin aging - contributors and inhibitors. J Cosmet Dermatol. 2018;17(2):124-137.

6. Panich U, Sittithumcharee G, Rathviboon N, Jirawatnotai S. Ultraviolet radiation-induced skin aging: the role of DNA damage and oxidative stress in epidermal stem cell damage mediated skin aging. Stem Cells Int. 2016;2016(1):1-14.

7. Puri P, Nandar S, Kathuria S, Ramesh V. Effects of air pollution on the skin: a review. Indian J Dermatol Venereol Leprol. 2017;83(4):415-423.

8. Zhang S, Duan E. Fighting against skin aging: the way from bench to bedside. Cell Transplant. 2018;27(5):729-738.

9. Ghersetich I, Lotti T, Campanile G, Grappone C, Dini G. Hyaluronic acid in cutaneous intrinsic aging. Int J Dermatol. 1994;33(2):119-122.

10. Fore J. A review of skin and the effects of aging on skin structure and function. Ostomy Wound Manage. 2006;52:24-35; quiz 36-37.

11. He W, Goodkind D, Kowal P. An ageing world: 2015 international population reports. Report number P95-16-1. United States Census Bureau; 2016. Available from: https://www.census.gov/content/dam/Census/ library/publications/2016/./p95-16-1.pdf. Accessed July 14, 2018.

12. asdsnet. ASDS consumer survey on cosmetic dermatologic procedures. American Society for Dermatologic Surgery; 2015. Available from: https://www.asds.net/_Media.aspx?id=8963. Accessed July 14, 2018.

13. Ang P, Barlow RJ. Nonablative laser resurfacing: a systematic review of the literature. Clin Exp Dermatol. 2002;27(8):630-635.

14. Attenello N, Maas C. Injectable fillers: review of material and properties. Facial Plast Surg. 2015;31(1):29-034.

15. Tyers AG. Brow lift via the direct and trans-blepharoplasty approaches. Orbit. 2006;25(4):261-265.

16. Wan D, Small KH, Barton FE. Face lift. Plast Reconstr Surg. 2015; 136(5):676e-689e.

17. Carruthers J, Carruthers A, Humphrey S. Introduction to fillers. Plast Reconstr Surg. 2015;136(5 Suppl):120S-131S.

18. Ascher B, Bayerl C, Brun P, et al. Efficacy and safety of a new hyaluronic acid dermal filler in the treatment of severe nasolabial lines - 6-month interim results of a randomized, evaluator-blinded, intra-individual comparison study. J Cosmet Dermatol. 2011;10(2):94-98.

19. Hong JY, Choi EJ, Choi SY, Li K, Kim BJ. Randomized, patient/ evaluator-blinded, intraindividual comparison study to evaluate the efficacy and safety of a novel hyaluronic acid dermal filler in the treatment of nasolabial folds. Dermatol Surg. 2018;44(4):542-548.

20. McCracken MS, Khan JA, Wulc AE, et al. Hyaluronic acid gel (Restylane) filler for facial rhytids: lessons learned from American Society of Ophthalmic Plastic and Reconstructive Surgery member treatment of 286 patients. Ophthalmic Plast Reconstr Surg. 2006;22(3):188-191.

21. Baumann L, Weiss RA, Grekin S, et al. Comparison of Hyaluronic Acid Gel With (HARDL) and Without Lidocaine (HAJUP) in the treatment of moderate-to-severe nasolabial folds: a randomized, evaluator-blinded study. Dermatol Surg. 2018;44(6):833-840.

22. Monheit G, Beer K, Hardas B, et al. Safety and effectiveness of the hyaluronic acid dermal filler VYC-17.5L for nasolabial folds: results of a randomized, controlled study. Dermatol Surg. 2018;44(5):670-678.
Clinical, Cosmetic and Investigational Dermatology

\section{Publish your work in this journal}

Clinical, Cosmetic and Investigational Dermatology is an international, peer-reviewed, open access, online journal that focuses on the latest clinical and experimental research in all aspects of skin disease and cosmetic interventions. This journal is included on PubMed. The manuscript management system is completely online

\section{Dovepress}

and includes a very quick and fair peer-review system, which is all easy to use. Visit http://www.dovepress.com/testimonials.php to read real quotes from published authors 